\title{
Laser Spot Center Detection and Comparison Test
}

\author{
Jun $\mathrm{ZHU}^{1^{*}}$, Zhengjie $\mathrm{XU}^{1}$, Deli FU ${ }^{1}$, and Cong $\mathrm{HU}^{2}$ \\ ${ }^{1}$ College of Electronic Engineering, Guangxi Normal University, Guilin 541004, China \\ ${ }^{2}$ Guangxi Key Laboratory of Automatic Detecting Technology and Instruments, Guilin University of Electronic \\ Technology, Guilin 541004, China \\ "Corresponding author: Jun ZHUＥ-mail: zhujun1985@gxnu.edu.cn
}

\begin{abstract}
High efficiency and precision of the pot center detection are the foundations of avionics instrument navigation and optics measurement basis for many applications. It has noticeable impact on overall system performance. Among them, laser spot detection is very important in the optical measurement technology. In order to improve the low accuracy of the spot center position, the algorithm is improved on the basis of the circle fitting. The pretreatment is used by circle fitting, and the improved adaptive denoising filter for TV repair technology can effectively improves the accuracy of the spot center position. At the same time, the pretreatment and de-noising can effectively reduce the influence of Gaussian white noise, which enhances the anti-jamming capability.
\end{abstract}

Keywords: Spot; TV repair technology; spot center

Citation: Jun ZHU, Zhengjie XU, Deli FU, and Cong HU, "Laser Spot Center Detection and Comparison Test," Photonic Sensors, 2019, 9(1): 49-52.

\section{Introduction}

The laser spot images still have various challenges. Although the center can be seen with naked eyes, it has not high accuracy. By physical means, the middle point of the spot can be accurately found, but it needs more time and requires a high degree of precision for the physical equipment. Relatively, faster positioning algorithm using software has higher speed and accuracy for finding the spot center position. High efficiency and precision of the pot center detection, which are the foundations of avionics instrument navigation and optics measurement basis for many applications, have a noticeable impact on the overall system performance. Reference [1] presented an algorithm of laser spot detection based on circle fitting with a high precision, which has a high degree of accuracy. Reference [2] compared circle fitting and traditional algorithm of laser spot position, and the algorithm of circle fitting has advantages of high precision and efficiency. Reference [3] proposed a new algorithm of the image filters, and its performance was proved to be also very good. Reference [4] presented an algorithm of adaptive median filtering, which could automatically find and remove cycle disturbances. Yet, the laser spot has noise and other interference in real optical measurement, resulting in uneven distribution of spot signal strength. In general, the above algorithm does not meet requirements of accuracy or speed, and its anti-jamming ability is poor [5-9]. Therefore, the improved algorithm is based on the original circle fitting. Before circle fitting, the algorithm has pretreatment to solve

Received: 18 May 2017/ Revised: 29 January 2018

(C) The Author(s) 2018. This article is published with open access at Springerlink.com

DOI: $10.1007 / \mathrm{s} 13320-018-0432-\mathrm{x}$

Article type: Regular 
various interferences, and then an improved TV filtering algorithm can have better filtering for Gaussian white noise and finally pinpoint the spot center.

\section{Theoretical analysis}

Fitting is based on least squares (smallest residual sum of squares) guidelines for mathematical methods [10-12]. By fitting the target coordinates or grayscale, you can get the form of continuous functions, so that you can determine the values that describe each parameter object [13-15]. When the target is characterized by round or ellipse, we extract a set of points of the target circle fitting, whose method is the laser beam profile.

The equation of a circle is as follows:

$$
(x-a)^{2}+(y-b)^{2}=r^{2} .
$$

At this point, take the residuals as follows:

$$
\varepsilon_{i}=\left(x_{i}-a\right)^{2}+\left(y_{i}-b\right)^{2}-r .
$$

Abovely, $a$ and $b$ represent a collection of all the boundaries, and $x_{i}$ represents a sharp point coordinates.

Residual sum of squares function is calculated as follows:

$$
Q=\sum_{i \in E} \varepsilon_{i}^{2}=\sum_{i \in E}\left[\left(x_{i}-a\right)^{2}+\left(y_{i}-b\right)^{2}-r^{2}\right]^{2} .
$$

By the least squares method, we can get the following equation:

$$
\frac{\partial Q}{\partial a}=\frac{\partial Q}{\partial b}=\frac{\partial Q}{\partial r}=0
$$

which is simplified by

$$
\begin{gathered}
a^{2}-2 \bar{x} a+b^{2}-2 \bar{y} b-r^{2}+\overline{x^{2}}+\overline{y^{2}}=0 \\
\bar{x} a^{2}-2 \overline{x^{2}} a+\bar{x} b^{2}-2 \overline{x y} b-\bar{x} r^{2}+\overline{x^{3}}+\overline{x y^{2}}=0 \\
\bar{y} a^{2}-2 \overline{x y} a+\bar{y} b^{2}-2 \overline{y^{2}} b-\bar{y} r^{2}+\overline{x^{2} y}+\overline{y^{3}}=0 .
\end{gathered}
$$

Parameters satisfy the following formula:

$$
\overline{x^{m} y^{n}}=\sum_{i \in E} x_{i}^{m} y_{i}^{n} / \sum_{i \in E} 1 \text {. }
$$

Then, we can get

$$
\left(\bar{x}^{2}-\overline{x^{2}}\right) a+(\overline{x y}-\overline{x y}) b=\frac{1}{2}\left(\overline{x^{2}} \bar{x}+\bar{x} \overline{y^{2}}-\overline{x^{3}}-\overline{x y^{2}}\right)
$$

$$
(\overline{x y}-\overline{x y}) a+\left(\bar{y}^{2}-\overline{y^{2}}\right) b=\frac{1}{2}\left(\overline{x^{2}} \bar{y}+\bar{y} \overline{y^{2}}-\overline{x^{2} y}-\overline{y^{3}}\right) .
$$

Lastly, we can combine the expression parameters with the formula parameters:

$$
\begin{gathered}
a=\frac{\left(\overline{x^{2}} \bar{x}+\bar{x} \overline{y^{2}}-\overline{x^{3}}-\overline{x y^{2}}\right)\left(\bar{y}-\overline{y^{2}}\right)-\left(\overline{x^{2}} \bar{y}+\bar{y} \overline{y^{2}}-\overline{x^{2} y}-\overline{y^{3}}\right)(\overline{x y}-\overline{x y})}{2\left(\bar{x}-\overline{x^{2}}\right)\left(-\bar{y}-\overline{y^{2}}\right)-2(\overline{x y}-\overline{x y})} \\
b=\frac{\left(\overline{x^{2}} \bar{y}+\bar{y} \overline{y^{2}}-\overline{x^{2} y}-\overline{y^{3}}\right)\left(\bar{x}^{2}-\overline{x^{2}}\right)-\left(\overline{x^{2}} \bar{x}+\bar{x} \overline{y^{2}}-\overline{x^{3}}-\overline{x y^{2}}\right)(\overline{x y}-\overline{x y})}{2\left(\bar{x}^{2}-\overline{x^{2}}\right)\left(\bar{y}^{2}-\overline{y^{2}}\right)-2(\overline{x y}-\overline{x y})^{2}} \\
r=\sqrt{a^{2}-2 \bar{x} a+b^{2}-2 \bar{y} b+\overline{x^{2}}+\overline{y^{2}}} .
\end{gathered}
$$

As can be seen by the above formulae, the algorithm is derived by the least squares circle fitting. Our algorithm can calculate each parameter, but its calculation speed is quick because it only calculates the central parameter.

\section{Test programme}

\subsection{Process}

The flow chart of the improved algorithm is shown in Fig. 2.

\subsection{Improved algorithms and performance analysis}

White noise is a constant power spectral density of random signals or stochastic process. In other words, the signal powers are the same in all frequency bands, as white light is made up of frequency (color) of light mixed together. This flat nature of the power spectrum of the signal is said to be "white", and thus this signal is called white noise. When being obtained from Gaussian distribution of the random sample values, samples consisting of random process is the "white Gaussian noise". 


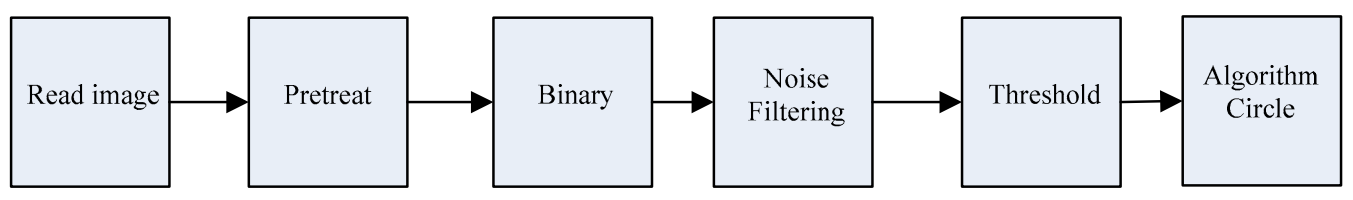

Fig. 2 Processes of improvement algorithm.

Firstly, we deal with the noise around image. Then, the improved TV filtering method is used to remove spots of Gaussian white noise. We test Gaussian white noise filtering and median (median filtering, $\mathrm{MF}$ ) in order to verify the effectiveness of adaptive TV. The results of comparison are shown in Fig. 3.
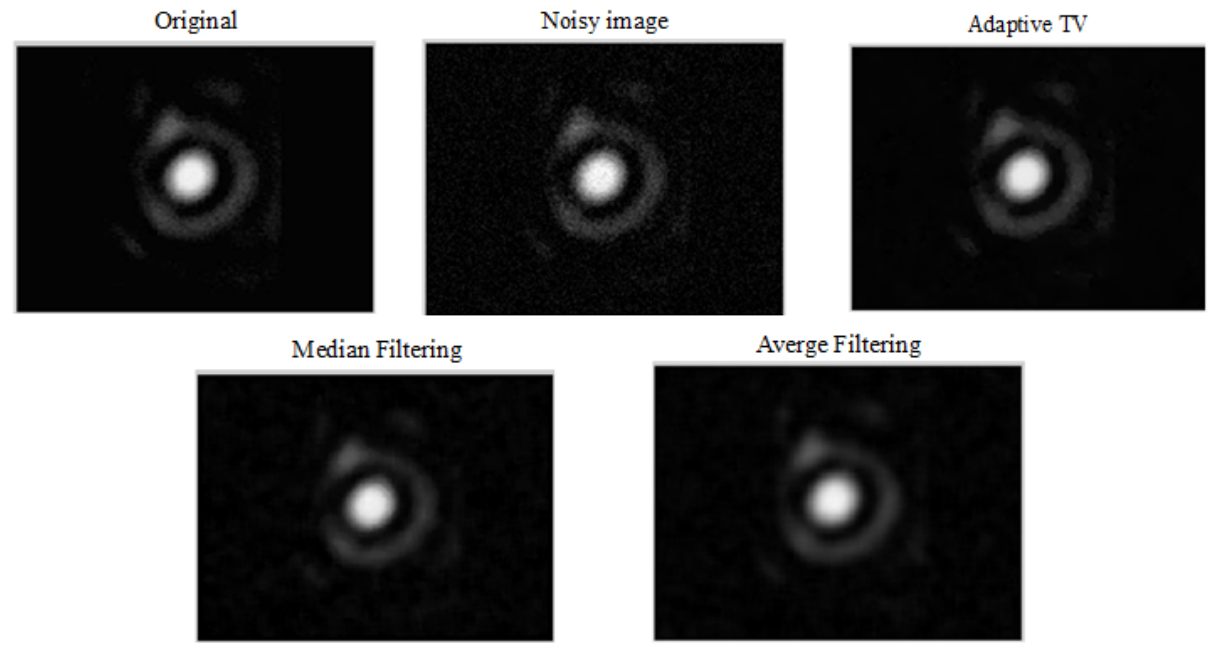

Fig. 3 Results of our algorithm.

\subsection{Results and analysis}

As we can see from Fig. 4, our method can better protect spot edge and traditional round edge in the process of detecting image. Also, we discover that the algorithm tends to lose some real information, which is not good for research of spot.

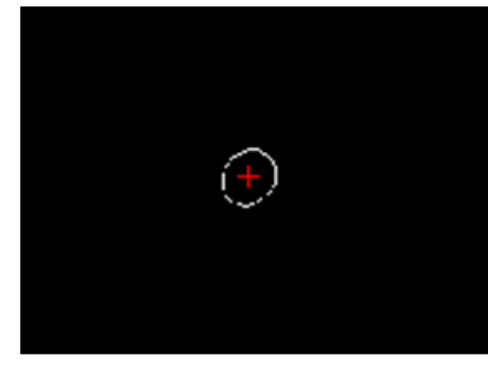

Fig. 4 Process of detecting image.

\section{Conclusions}

Based on the experimental results, we can conclude that the improved algorithm of laser spot center detection on circle fitting is better than the original method. The performance of the improved algorithm for noise immunity is better with the
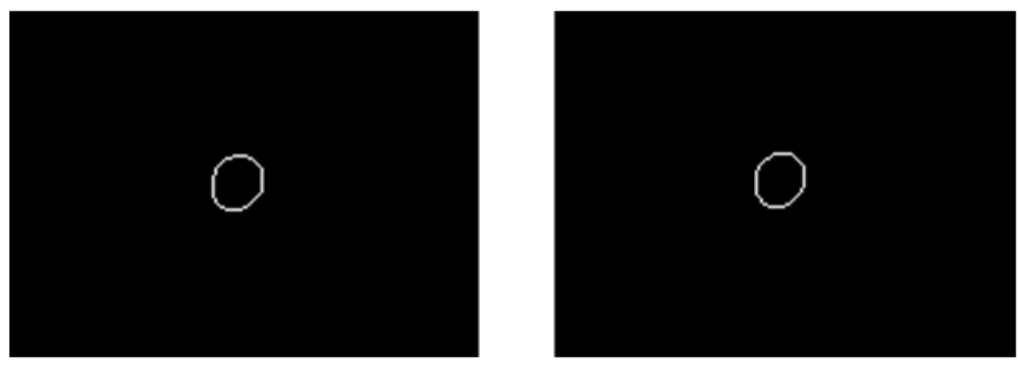

high accuracy. Therefore, studying on the algorithm of follow-up research on the spot is important.

\section{Acknowledgment}

This work was supported by Guangxi Natural Science Foundation (Grant No. 2017GXNSFAA198261), Guangxi Normal 
University Key Program (Grant No. 2015ZD03), and Innovation Project of Guangxi Graduate Education (Grant Nos. XJGY201807 and XJGY201811), and Guangxi Scholarship Fund of Guangxi Education Department (Grant No.2017).

OpenAccess This article is distributed under the terms of the Creative Commons Attribution 4.0 International License (http://creativecommons.org/licenses/by/4.0/), which permits unrestricted use, distribution, and reproduction in any medium, provided you give appropriate credit to the original author(s) and the source, provide a link to the Creative Commons license, and indicate if changes were made.

\section{References}

[1] Z. K. Wu, G. K. Li, W. T. Wang, X. Yang, X. J. Tang, and D. S. Jiang, "Algorithm of laser spot detection based on circle fitting," Infrared and Laser Engineering, 2002, 36(3): 276-279.

[2] B. Kong, Z. Wang, and Y. S. Tan, "Algorithm of laser spot detection based on circle fitting," Infrared and Laser Engineering, 2002, 36(3): 276-279.

[3] B. Jiang and Z. Rahman, "Noise reduction using multi-resolution edge analysis," SPIE, 2009, 7245: 724507-1-724507-11.

[4] A. R. Weeks, T. Kasparis, and B. Kief, "Adaptive median filter for the removal of periodic interference from an image," SPIE, 1994, 2238(022): 207-217.

[5] J. Bigeon, S. L. Liepvre, S. Vassant, N. Belabas, N. Bardou, C. Minot, et al., "Strong coupling between self-assembled molecules and surface plasmon polaritons," Journal of Physical Chemistry Letters, 2017, 8(22): 5626-5632.

[6] F. Y. Gan, Y. J. Wang, C. W. Sun, G. R. Zhang, H. Y. Li, J. J. Chen, et al., "Widely tuning surface plasmon polaritons with laser-induced bubbles," Advanced Optical Materials, 2017, 5(4): 1600545-1-1600545-7.

[7] W. Wang, S. S. Gao, and Y Meng, "Transmission characteristics of surface plasmon polaritons in ' $\theta$ '-shaped resonator,' Acta Physica Sinica, 2017, 66: 017301-1-017301-5.

[8] Y. Q. Yang, R. Shi, X. N. Yu, and T. N. Gao, “An algorithm to raise the locating precision of laser spot center based on Hough transform," Acta Optica Sinica, 1999, 19(12): 1655-1660.

[9] J. W. Cui, J. B. Tan, L. Ao, and W. J. Kang, "Optimized algorithm of laser spot center location in strong noise," Journal of Physics: Conference Series, 2005, 13(1): 312-315.

[10] T. Hashimoto, F. Yagami, M. Owada, T. Sugawara, and M. Kawamura, "Salt preference according to a questionnaire vs. dietary salt intake estimated by a spot urine method in participants at a health check-up center," Internal Medicine, 2008, 47(5): 399-403.

[11] L. L. Wang, Z. W. Hu, and H. X. Ji, "Laser spot center location algorithm based on Gaussian fitting," Journal of Applied Optics, 2012, 33(05): 985990.

[12] J. W. Cui, J. B. Tan, L. Ao, and W. J. Kang, "Optimized algorithm of laser spot center location in strong noise," Journal of Physics, 2005, 13: 312-315.

[13] Y. M. Xu, C. Xing, and G. L. Liu, "Comparisons of several methods of laser spot center detection," Hydrographic Surveying \& Charting, 2007, 27(2): 74-76.

[14] Z. L. Lan and X. F. Yang, "Practical improvement of laser spot center location algorithm," Computer Engineering, 2008, 34(6): 7-9.

[15] Y. Zheng, "Subpixel location of the center of laser spot based on spatial moments," Laser \& Infrared, 2005, 7: 521-523. 